\title{
Spatio-temporal Reasoning for Vague Regions
}

\author{
Zina M. Ibrahim and Ahmed Y. Tawfik \\ University of Windsor, 401 Sunset Avenue, \\ Windsor, Ontario N9B 3P4, Canada \\ \{ibrahim, atawfik\}@uwindsor.ca
}

\begin{abstract}
This paper extends a mereotopological theory of spatiotemporal reasoning to vague "egg-yolk" regions. In this extension, the egg and its yolk are allowed to move and change over time. We present a classification of motion classes for vague regions as well as composition tables for reasoning about moving vague regions. We also discuss the formation of scrambled eggs when it becomes impossible to distinguish the yolk from the white and examine how to incorporate temporally and spatially dispersed observations to recover the yolk and white from a scrambled egg. Egg splitting may occur as a result of the recovery process when available information supports multiple egg recovery alternatives. Egg splitting adds another dimension of uncertainty to reasoning with vague regions.
\end{abstract}

\section{Introduction}

Spatiotemporal knowledge representations proposed so far include qualitative models of kinematics [14] as well as many spatiotemporal logics [9]11. These logics augment a temporal logic with a spatial one. For example, temporal modal logic and RCC-8 are combined in [17 to represent and reason about spatiotemporal change.

Muller's theory [11|12,13] describes the relations among spatiotemporal objects via a primitive of connectedness. As humans usually reason with imprecise or incomplete information, the notion of vague regions of space-time has attracted some attention [6]. This paper extends Muller's theory to vague regions using the egg-yolk theory [5] as a base.

Due to the importance of space in AI, lot of work has been put into formulating representations of space in the past decade. Also, because systems in AI usually deal with imprecise, incorrect or incomplete information, qualitative models have been the preferred choice as opposed to quantitative ones. In particular, Qualitative Spatial Representation (QSR) models have flourished and are accepted as the models that take as ontological primitives extended regions of space as opposed to points. One such theory is the Region Connectedness Calculus (RCC), which is a well-known first-order theory that deals with regions in a topological manner [15. The theory's original intension has been for regions with precise boundaries, or in other words crisp regions of space. However, it was extended to deal with vague regions in [45110] and formed what is known as the 
egg-yolk theory that represents a vague region of space by two crisp regions, the egg and the yolk. Whereas the yolk represents the objects that definitely belong to the region, the white is for the objects that may or may not belong to the region. The objects outside the egg definitely do not belong to the region.

Qualitative theories of time have also had a rich history starting with Allen's interval logic [1] that has paved the way to reason with time in a qualitative manner.

Due to the richness in qualitative theories of both space and time, Qualitative spatiotemporal reasoning was the next natural step. Work in this direction was also motivated by the need to model motion in a qualitative fashion as the usual quantitative methods are not favored in AI. Work in this direction includes Muller's work 1112 13. which is a mereotopological theory of space-time inspired by [2. The theory has as primitives extended regions of space-time; it defines all objects made of this primitive to be occurants and views them as space-time histories. The relations between the regions (histories) are based on the temporal and spatial relationships that hold among them, and a set of axioms supports spatiotemporal inference from spatial and temporal information. The theory combines RCC-8 spatial representation and Allen's interval logic. It further defines motion by examining the relationships between histories at two consecutive time intervals and accordingly identifies classes of motion that may be true as regions move 11 .

In this paper, we present the building blocks to extending Muller's theory to vague regions (histories) of space-time using the egg-yolk representation. We also aim at constructing a set of motion classes similar to those of Muller found in [1] for vague regions and utilize composition tables to obtain the possible relations between two (or more) moving vague regions. Our approach is partly inspired by the scrambled-egg theory [6,7] in extending the egg-yolk theory from dealing with spatial regions to dealing with regions of space-time. Guesgen's work on scrambled eggs suggests that as regions start to move, less information becomes available regarding their yolk and white, and hence the parthood between the yolk and the egg can no longer be assumed, and a scrambled egg results. Here we examine the possibility of recovering the egg-yolk structure from a scrambled egg by incorporating fixed-interval object re-identification [16]. Object re-identification uses recent observations to reorganize egg-yolk pairs, thus reducing the uncertainty. More specifically, we identify the weaknesses of the eggyolk theory in the spatiotemporal domain and suggest a solution for overcoming this weakness.

\section{Overview of Muller's Spatiotemporal Theory}

The primitive entities in Muller's theory are spatiotemporal regions. The relations among these regions can be spatiotemporal as well as temporal. A spatiotemporal relationship describes the spatial motion of a region over time with respect to a static reference region, while the temporal relationship between two regions describes how the two spatiotemporal evolutions are related in time. 
The theory follows a general trend in spatiotemporal representation by combining a spatial theory as a topological base, along with a temporal theory and accordingly formulating the spatiotemporal interactions between the two. The RCC- 8 set is chosen as the topological base due to its wide acceptance and inherit conceptual neighborhood structure. The temporal relations are those of Allen [1. The resulting theory is mereotopological and is based on assumptions compatible with RCC-8. The theory does not perceive points as primitives and relies on intervals/regions in representing spatial/temporal quantities. The choice of Allen's interval logic and RCC- 8 produces a coherent spatiotemporal theory from this perspective.

The spatial extents of two regions $x$ and $y$ are connected $(C x y)$ if the distance between them is zero. The same is also true for the temporal connection between two intervals $a$ and $b$ denoted by $(a \diamond b)$. The axiom given in 1 captures the relationship between spatiotemporal connectedness and temporal connectedness.

$$
C x y \rightarrow x \diamond y
$$

Note that for notational clarity we adopt the following convention:

- Spatiotemporal operators precede the regions they operate upon (prefix notation)

- Temporal operators come between the regions they operate upon (infix notation)

The axiom given in (1) is the base for all other types of interaction between the topological (spatial) and temporal aspects of the theory. Another important concept in Muller's theory is the notion of a 'temporal slice' denoted by $(x \mid y)$, which refers to the subinterval of $x$ corresponding to the "lifetime" of $y$ when $y$ $\subseteq_{t} x[1]$.

$x \mid y$ is a continuous section of the trajectory of region $x$ such that $y \subseteq_{t} x$.

TEMP_IN $x y$ describes the notion of temporary parthood. The following defines the meaning of region $x$ being a part of region $y$ temporarily.

$$
T E M P_{-} I N x y \text { if } \exists z, u:(z \mid x \wedge P z y) \wedge\left(u \mid x \wedge\left(u \subseteq_{t} y\right) \wedge \neg C u y\right) .
$$

Classes of motion are identified and formally defined according to the possible interactions between spatiotemporal objects. For example, region $x$ is said to have reached region $y$ during an interval $z$ if $x$ finishes $y$ and $x$ is temporarily in $y$.

$$
R E A C H z x y \text { if TEMP_IN } x|z y| z \wedge(x|z \cdot y| z \text { FINISHES } z) .
$$

In (4), FINISHES is based on Allen's interval logic and is defined as:

$$
x \text { FINISHESy if } x \subseteq_{t} y \wedge \forall z(x<z \rightarrow y<z) \wedge \neg y \subseteq_{t} x .
$$

Where $<$ represents a temporal precedence relationship. 
The symmetric motion class LEAVE is obtained by replacing FINISHES by STARTS, defined in (6) to finally have the definition given in (7).

$$
\begin{gathered}
x S T A R T S y \text { if } x \subseteq_{t} y \wedge \forall z(z<x \rightarrow z<y) \wedge \neg y \subseteq_{t} x . \\
L E A V E z x y \text { if TEMP_IN } x|z y| z \wedge(x|z \cdot y| z S T A R T S z) .
\end{gathered}
$$

Note that this definition of LEAVE assumes that the smaller region leaves the larger region and cannot be used to capture statements like "Leaving a book" but works for statements like "Leaving town".

The motion of region $x$ is said to be internal in region $y$ during interval $z$ if all time slices of $x$ are spatial proper parts of corresponding time slices of region $y$ throughout the interval $z$.

$$
\text { INTERNAL zxy if } P P x|z y| z .
$$

The motion of region $x$ is external to region $y$ throughout the interval $z$ if at no time during $z$ has $x$ been connected to $y$.

$$
\text { EXTERNAL zxy if } \neg C x|z y| z .
$$

Region $x$ is considered to have hit region $y$ if the two regions became externally connected at the end of interval $z$.

$$
\begin{gathered}
H I T z x y \text { if } E C x|z y| z \wedge \forall x_{1}, y_{1}\left[\left(P x_{1} x\left|z \wedge P y_{1} y\right| z \wedge E C x_{1} y_{1}\right) \rightarrow\right. \\
\left.x_{1} \text { FINISHES } z \wedge y_{1} \text { FINISHES }\right] .
\end{gathered}
$$

Region $x$ may also split into two subregions during the interval $z$

$$
\begin{gathered}
S P L I T z x y \text { if } E C x|z y| z \wedge \forall x_{1}, y_{1}\left[\left(P x_{1} x\left|z \wedge P y_{1} y\right| z \wedge E C x_{1} y_{1}\right) \rightarrow\right. \\
\left.x_{1} S T A R T S z \wedge y_{1} S T A R T S z\right] .
\end{gathered}
$$

We can use the above motion classes to defined more complex classes. For example, a region $x$ has crossed another region $y$ during an interval $z$ if $x$ reaches $y$ and leaves it in two consecutive subintervals of $z$.

$$
\begin{aligned}
& C R O S S z x y \text { if } \exists z_{1}, z_{2}\left(z=z_{1}+z_{2} \wedge z_{1} M E E T S z_{2} \wedge R E A C H z_{1} x y\right. \\
& \left.\wedge L E A V E z_{2} x y\right) \text {. }
\end{aligned}
$$

Where:

$$
x M E E T S y \text { if } x<y \wedge x \diamond y .
$$

\section{Extending Muller's Theory to Vague Egg-Yolk Regions}

One of the limitations of Muller's theory as presented in section 2 is that it assumes that the spatiotemporal regions have clearly defined boundaries in both time and space. In many practical situations, it is not possible to represent crisp boundaries for regions in space. For example, it is not possible to precisely represent the boundaries of an ocean, or a mountain. The boundaries of a city 
or even a property can also be unclear or unknown at a high resolution. To reason about a spatiotemporal mobile region, it is completely unreasonable to assume crisp deterministic motion unless the region's location is continuously and accurately monitored. In spatiotemporal reasoning, spatial and temporal vagueness pervade.

The egg-yolk theory [5] has been introduced to handle spatial vagueness. According to this theory, a region in space with vague boundaries is defined in terms of two crisp regions the egg and the yolk. The vague boundaries may lies anywhere in the egg-white between the yolk and the shell of the egg. The yolk represents the region in space that definitely belongs to the region and any region in space outside the egg is certainly outside the region. Figure 1 shows all the 46 possible topological relations between two egg-yolk regions.

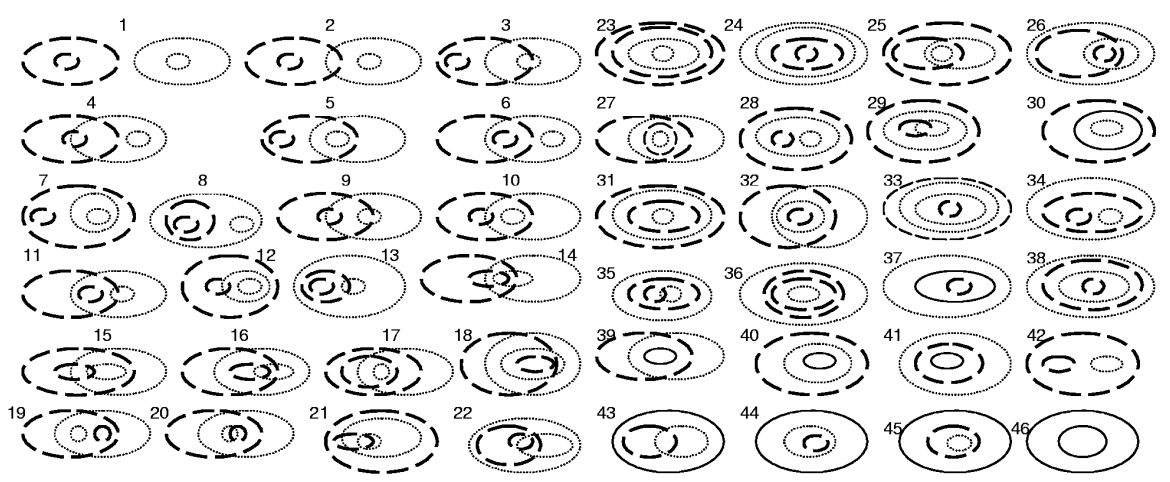

Fig. 1. The 46 Egg-Yolk Relationships

Some of the spatial relationships defined for regions with crisp boundaries in RCC-8 are no longer valid for egg-yolk region. For example, it is not generally correct to describe two egg-yolk regions as externally connected (EC) or tangential proper part (TPP) of one another. 4] has presented a set of five spatial relationships (RCC-5) that may hold between two spatial regions including egg-yolk regions. Given two crisp regions $x$ and $y$ with a set of RCC- 8 relations holding between them, if $x$ and $y$ become vague, is it now possible to deduce the set of egg-yolk relations that hold between the vague versions of the two regions using the RCC-5 set shown in Figure 2 along with the RCC-8 set.

\subsection{Mapping RCC-8 to RCC-5}

Here, we axiomatize a conversion process used in [4] to obtain RCC-5 from RCC-8 and back, as shown in figure 2 ,

The axiom given in (14) states that regions $x$ and $y$ are distinct regions (non-overlapping) if they are disconnected or externally connected.

$$
D R x y=D C x y \vee E C x y \text {. }
$$




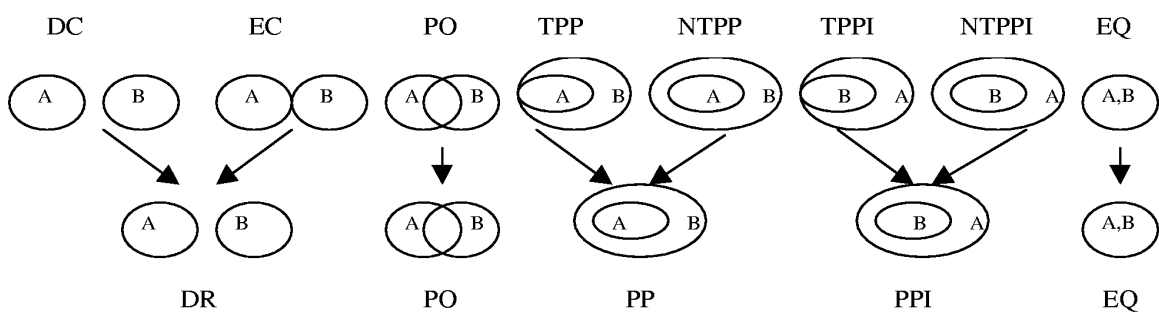

Fig. 2. The RCC-8 and RCC-5 Relations

The axiom given in (15) states that region $x$ is a proper part of region $y$ if it is a tangent proper part or a non-tangent proper part.

$$
P P x y=T P P x y \vee N T P P x y .
$$

The axiom given in (16) provides the inverse relationships to the previous axiom.

$$
\text { PPI } x y=T P P I x y \vee N T P P I x y .
$$

The relations EC, TPP and TPPI are based on the exact location of the region boundaries and therefore cannot be defined for a region with vague boundaries. Hence, the exioms given in (17:19) describe impossible situations.

$$
\begin{aligned}
& \neg \exists x, y E C x y \wedge(V A G U E x \wedge V A G U E y) . \\
& \neg \exists x, y T P P x y \wedge(V A G U E x \wedge V A G U E y) . \\
& \neg \exists x, y T P P I x y \wedge(V A G U E x \wedge V A G U E y) .
\end{aligned}
$$

As a result, the relations EC, TPP, TPPI do not have vague counterparts. Given that $x$ and $y$ are vague regions, axioms given in (14!16) become:

$$
\begin{gathered}
D R x y=D C x y . \\
P P x y=N T P P x y . \\
P P I x y=N T P P I x y .
\end{gathered}
$$

\subsection{Clustering the EGG-YOLK Relations}

The egg-yolk relations shown in Figure 1, which is taken from [5] can be clustered according to the RCC-5 relation that hold between their outer boundaries (boundaries of their eggs) as given in Table1. The table provides a direct method for finding the relations between the vague versions of two crisp regions $x$ and $y$ having a set of RCC-5 relations holding between them. We add the subscript $v$ to the name of the RCC-5 relations to emphasize that the name given is merely a representation of the possible Egg-Yolk relations that share a common RCC-5 relation between their eggs. 
Table 1. Clustering the Egg-yolk Relations

\begin{tabular}{|c|l|}
\hline RCC-5(x,y) & Egg-Yolk Group \\
\hline $\mathrm{DR}_{v}$ & 1 \\
\hline $\mathrm{PO}_{v}$ & 2345691011141517181920273239 \\
\hline $\mathrm{EQ}_{v}$ & 4243444546 \\
\hline $\mathrm{PP}_{v}$ & 813222426343536373841 \\
\hline $\mathrm{PPI}_{v}$ & 7122123252829303133 \\
\hline
\end{tabular}

As a side note, Figure 1 supports the correctness of the axioms given in (20), (21) and (22). For example, examining the egg-yolk relations that correspond to the RCC- 5 relation DR, there is only one case. In this case, the eggs are disconnected (DC) and no case of the eggs related via EC, which satisfies (20). The same reasoning can be used to verify (21) with PP and (22) with PPI.

\section{General Algorithm}

The transformations built in the prvious discussions can be formulated as an algorithm that, given two regions $x$ and $y$ and the RCC- 8 relations that hold between them, generates a list of all possible egg-yolk pairs given the RCC-8 list.

\section{Algorithm Convert}

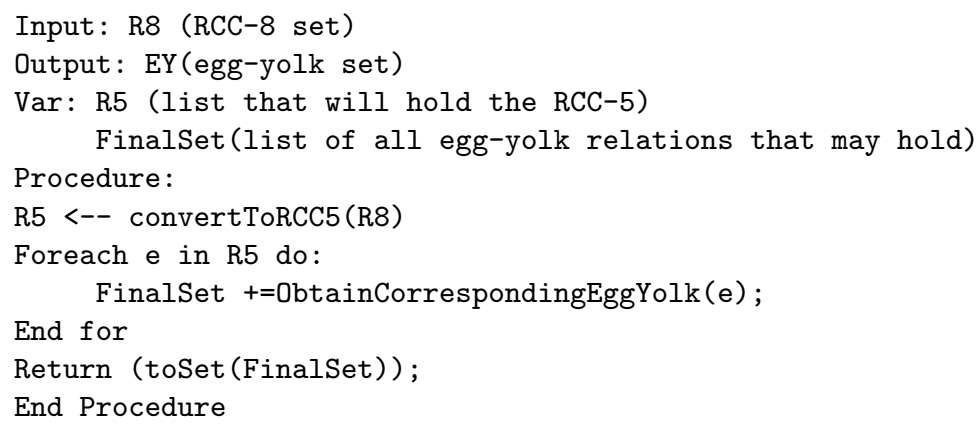


End for

Return (toList (R5));

End Procedure

\subsection{Motion and Vague Regions}

[13] defines the following classes of motion: LEAVE, REACH, CROSS, HIT, INTERNAL, EXTERNAL, and SPLIT. Constructing motion classes for vague regions is different as the class definition must not rely on the fact that the regions have well-defined boundaries. However, by assuming that we have enough information to discern the egg and the yolk for every subinterval throughout the motion, it is possible to redefine the classes of motion for vague regions. This assumption is equivalent to defining a vague time slice denoted by $x / y$ as a continuous portion of the trajectory of region $x$ that occurs during the lifetime of region $y$ provided that yolk $x$, white $x$, yolk $y$ and white $y$ are distinct regions throughout the time slice.

$x / y$ is continuous section of the trajectory of vague region $x$ such that

$x \subseteq_{t} y \wedge D R$ yolk $x$ white $x \wedge D R$ yolk $y$ white $y$ throughout $x / y$.

Using the definition for the vague time slice, we can define classes of motion for LEAVE, REACH, CROSS, INTERNAL and EXTERNAL. As the definitions provided in Section 2 for these classes of motion do not rely on knowing the exact boundaries of the region, we can retain the same definitions after restricting them as follows.

$$
\begin{gathered}
R E A C H_{v} z x y \text { if } R E A C H z x \text { yolky. } \\
L E A V E_{v} z x y \text { if } L E A V E z x \text { yolky. } \\
I N T E R N A L_{v} z x y \text { if INTERNALzx yolky. } \\
\text { EXTERNAL } z x y \text { if EXTERNALzx yolky. } \\
\text { CROSS }_{v} z x y \text { if CROSS zx yolky. }
\end{gathered}
$$

Where $x$ and $y$ denote the whole region including the vague boundaries, yolk $y$ denotes the region definitely inside the vague boundaries and white $y$ is the vague part of the region.

However, a HIT is not possible to define for vague regions as the intuitive definition relies on external connection between the outer boundaries of the regions in motion. This is obviously not possible for vague regions as their outer boundaries are fuzzy. It is possible to construct an alternative definition suitable for vague regions. We define a POSSIBLE_HIT between two vague regions as the motion class that holds when the yolk part of the two regions overlap.

$$
\begin{aligned}
& \text { POSSIBLE_HIT zxy if } P O_{v} \text { yolk } x \mid z \text { yolk } y \mid z \wedge \forall x_{1}, y_{1}\left[\left(P x_{1} \text { yolk } x \mid z\right.\right.
\end{aligned}
$$

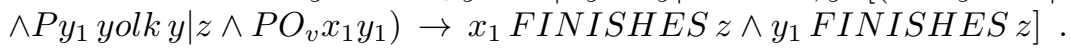


The effect of vagueness is not confined to HIT. The vagueness of the boundaries may necessitate that we define a POSSIBLE_REACH to describe the situation when region $x$ reaches the white of region $y$.

$$
\text { POSSIBLE_REACH zxy if REACH zxwhite } y \text {. }
$$

Similarly, a POSSIBLE_LEAVE occurs when region $x$ departs from the white of region $y$.

The motion of region $x$ is POSSIBLE_INTERNAL to region $y$ if it is internal to the egg but not confined to the yolk of region $y$.

$$
\begin{gathered}
\text { POSSIBLE_INTERNALzxy if INTERNALzxy^ } \\
\neg I N T E R N A L z x \text { yolky . }
\end{gathered}
$$

The motion of region $x$ is POSSIBLE_EXTERNAL to region $y$ if it is external to yolk $y$ but not to the whole egg $y$.

$$
\begin{gathered}
\text { POSSIBLE_EXTERNALzxy if EXTERNALzxyolky^ } \\
\neg E X T E R N A L z x y .
\end{gathered}
$$

Region $x$ is considered to have possibly crossed region $y$ if it had possibly reached it and possibly left it in two consecutive time intervals.

$$
\begin{aligned}
& \text { POSSIBLE_CROSS } z x y \text { if } \exists z_{1}, z_{2}\left(z=z_{1}+z_{2}\right) \wedge z_{1} M E E T S z_{2} \\
& \wedge \text { POSSIBLE_REACH } z_{1} x y \wedge \text { POSSIBLE_LEAVE } z_{2} x y .
\end{aligned}
$$

We have intentionally left the discussion of SPLIT to Section 5 as it is more appropriate to examine it along with the issues related to scrambled eggs.

\section{Reasoning about Motion}

Knowing that two spatiotemporal regions $u$ and $w$ are moving with respect to one another according to one of the motion classes during a certain temporal interval allows us to infer a set of spatial relationships that may hold during another interval. For example, knowing that region $u$ is leaving region $w$ during interval $z$ would allow us to conclude that one of the following three spatial relationship may hold during an interval $y$ that overlaps the interval $z$ as shown in figure 3 .

- Object $u$ starts off as a part of $w\left(P P_{v} u w\right)$

- Object $u$ can still partially overlap $w\left(P O_{v} u w\right)$

- Object $u$ can be completely outside $w\left(D R_{v} u w\right)$.

Therefore the temporally ordered spatial sequence $\left[\mathrm{PP}_{v} u w, \mathrm{PO}_{v} u w, \mathrm{DR}_{v}\right.$ $u w]$ corresponds to a $\mathrm{LEAVE}_{v}$ motion. As $\mathrm{REACH}_{v}$ is the opposite of $\mathrm{LEAVE}_{v}$, the temporally ordered sequence describing $\mathrm{REACH}_{v}$ is $\left[\mathrm{DR}_{v} u w, \mathrm{PO}_{v} u w, \mathrm{PP}_{v}\right.$ $u w]$. 

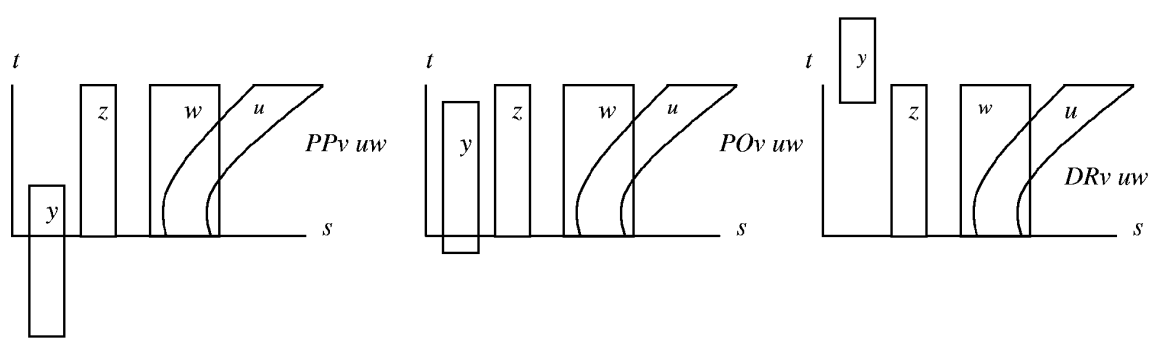

Fig. 3. The Three Possibilities for LEAVE

Similarly, a POSSIBLE_HIT can be described by the sequence [DR $\mathrm{DR}_{v} u w$, $\left.\mathrm{PO}_{v} u w\right]$. Other motion classes such as $\mathrm{INTERNAL}_{v}$ and EXTERNAL $v$ can be captured by the singleton spatial relationship $\left[\mathrm{PP}_{v} u w\right]$ and $\left[\mathrm{DR}_{v}\right]$ respectively. CROSS can be obtained by concatenating the sequences for $\mathrm{REACH}_{v}$ and $\mathrm{LEAVE}_{v}$ to obtain $\left[\mathrm{DR}_{v} u w, \mathrm{PO}_{v} u w, \mathrm{PP}_{v} u w, \mathrm{PO}_{v} u w, \mathrm{DR}_{v} u w\right]$. The sequences for POSSIBLE_LEAVE, POSSIBLE_REACH, POSSIBLE_INTERNAL, and POSSIBLE_EXTERNAL are identical to the sequences for LEAVE $\mathrm{REACH}_{v}, \mathrm{INTERNAL}_{v}$, and EXTERNAL $v$ respectively.

Frequently, we have information describing what happens over a time interval and we want to make deduction relative to another interval. For example, if we know that the motion of $u$ with respect to $w$ during $z$ is $\operatorname{INTERNAL}_{v} z u w$, then it is possible to deduce INTERNAL $z u w$ during any $x$ DURING $z$. If instead we are given $\operatorname{LEAVE}_{v} z u w$, then our conclusion becomes INTERNAL $\operatorname{IN}_{v}$ xu $\mathrm{V}$ $\operatorname{LEAVE}_{v} x u w \vee \mathrm{EXTERNAL}_{v} x u w$. In general we cannot make conclusions about intervals before or after the motion.

To generalize, assume that a temporal relationship $\mathrm{R}_{t}$ holds between intervals $x$ and $z$, and that region $u$ makes a motion of class $M$ relative to region $v$ over interval $z$, what spatial relationships hold over interval $x$ ? Table 2 answers this question for the classes of motions we discussed and eleven of Allen's thirteen relationships as it is not possible to make any conclusions for BEFORE and AFTER. Note that POSSIBLE_LEAVE, POSSIBLE_REACH, POSSIBLE_INTERNAL, and POSSIBLE_EXTERNAL behave in the same fashion as $\mathrm{LEAVE}_{v}, \mathrm{REACH}_{v}, \mathrm{INTERNAL}_{v}$, and EXTERNAL $v$ respectively.

Table 3 on the other hand, answers a different question. Assuming that region $u$ is moving with respect to region $v$ and that region $v$ has a static spatial relation $\mathrm{R}_{s}$ with respect to region $w$, then what can be deduce about region $u$ with respect to region $w$.

\section{Scrambled Eggs and Region Splitting}

Section 4 assumes that it is possible to distinguish the yolk from the white at all times. This assumption is rather unrealistic in some situations. As the object starts moving, it often becomes impossible to distinguish the white from the yolk. 
Table 2. Composing Motion with Temporal Information

\begin{tabular}{|c|c|c|c|c|c|c|}
\hline Motion $\mathrm{R}_{t}$ & $\mathrm{LEAVE}_{v}$ & $\begin{array}{c}\text { POSSIBLE }_{-} \\
\mathrm{HIT}_{v}\end{array}$ & $\mathrm{REACH}_{v}$ & $\mathrm{CROSS}_{v}$ & INTERNAL $_{v}$ & EXTERNAL $_{v}$ \\
\hline MEET & \multirow{3}{*}{$\begin{array}{l}\mathrm{PO}_{v} \\
\mathrm{PP}_{v}\end{array}$} & \multirow[t]{2}{*}{$\mathrm{DR}_{v}, \mathrm{PO}_{v}$} & \multirow{3}{*}{$\begin{array}{l}\mathrm{DR}_{v} \\
\mathrm{PO}_{v}\end{array}$} & \multirow{3}{*}{$\begin{array}{l}\mathrm{DR}_{v}, \\
\mathrm{PO}_{v}\end{array}$} & $\mathrm{PP}_{v}$ & \multirow[t]{2}{*}{$\mathrm{DR}_{v}, \mathrm{PO}_{v}$} \\
\hline $\mathrm{Ot}$ & & & & & $\mathrm{PO}_{v}$ & \\
\hline START & & \multirow[t]{2}{*}{$\mathrm{DR}_{v}$} & & & \multirow[t]{4}{*}{$\mathrm{PP}_{v}$} & \multirow[t]{4}{*}{$\mathrm{DR}_{v}$} \\
\hline DURING & All & & All & All & & \\
\hline$=\mathrm{t}$ & $\mathrm{PO}_{v}$ & \multirow[t]{2}{*}{$\mathrm{PO}_{v}$} & $\mathrm{PO}_{v}$ & $\mathrm{PO}_{v}$ & & \\
\hline FINISH & \multirow{2}{*}{$\begin{array}{l}\mathrm{DR}_{v}, \\
\mathrm{PO}_{v}\end{array}$} & & \multirow{2}{*}{$\begin{array}{l}\mathrm{PP}_{v} \\
\mathrm{PO}_{v}\end{array}$} & \multirow{2}{*}{$\begin{array}{l}\mathrm{DR}_{v} \\
\mathrm{PO}_{v}\end{array}$} & & \\
\hline $\mathrm{MEET}_{i}$ & & \multirow[t]{4}{*}{$\mathrm{PO}_{v}$} & & & \multirow[t]{4}{*}{$\mathrm{PO}_{v}, \mathrm{PP}_{v}$} & \multirow[t]{4}{*}{$\mathrm{DR}_{v}, \mathrm{PO}_{v}$} \\
\hline FINISH $_{i}$ & \multirow[t]{3}{*}{$\mathrm{PO}_{v}$} & & \multirow[t]{3}{*}{$\mathrm{PO}_{v}$} & \multirow[t]{3}{*}{$\mathrm{PO}_{v}$} & & \\
\hline START $_{i}$ & & & & & & \\
\hline DURING $_{i}$ & & & & & & \\
\hline
\end{tabular}

Table 3. Composing Motion with Spatial Information

\begin{tabular}{|c|c|c|c|c|c|c|}
\hline $\begin{array}{c}\text { Motion } \\
\mathrm{R}_{s}\end{array}$ & $\operatorname{LEAVE}_{v}$ & $\begin{array}{c}\text { POSSIBLE }_{-} \\
\mathrm{HIT}_{v}\end{array}$ & $\mathrm{REACH}_{v}$ & $\operatorname{CROSS}_{v}$ & $\operatorname{INTERNAL}_{v}$ & EXTERNAL $_{v}$ \\
\hline $\mathrm{DR}_{v}$ & $\begin{array}{l}\mathrm{DR}_{v} \\
\mathrm{PO}_{v}\end{array}$ & $\begin{array}{l}\mathrm{DR}_{v} \\
\mathrm{PO}_{v} \\
\mathrm{PPI}_{v}\end{array}$ & $\begin{array}{l}\mathrm{DR}_{v} \\
\mathrm{PO}_{v}\end{array}$ & $\begin{array}{l}\mathrm{DR}_{v} \\
\mathrm{PO}_{v}\end{array}$ & $\mathrm{DR}_{v}$ & All \\
\hline $\mathrm{PO}_{v}$ & $\begin{array}{c}\mathrm{DR}_{v} \\
\mathrm{PO}_{v} \\
\mathrm{PP}_{v}\end{array}$ & $\begin{array}{c}\mathrm{DR}_{v} \\
\mathrm{PO}_{v} \\
\mathrm{PP}_{v}\end{array}$ & $\begin{array}{c}\mathrm{DR}_{v} \\
\mathrm{PO}_{v} \\
\mathrm{PP}_{v}\end{array}$ & All & All & All \\
\hline $\mathrm{PP}_{v}$ & $\begin{array}{l}\mathrm{PP}_{v} \\
\mathrm{PO}_{v}\end{array}$ & $\begin{array}{l}\mathrm{PP}_{v} \\
\mathrm{PO}_{v}\end{array}$ & $\begin{array}{l}\mathrm{PP}_{v} \\
\mathrm{PO}_{v}\end{array}$ & $\begin{array}{l}\mathrm{PP}_{v} \\
\mathrm{PO}_{v}\end{array}$ & $\mathrm{PP}_{v}$ & All \\
\hline $\mathrm{PPI}_{v}$ & $\begin{array}{l}\mathrm{DR}_{v} \\
\mathrm{PO}_{v}\end{array}$ & $\begin{array}{l}\mathrm{PO}_{v} \\
\mathrm{DR}_{v}\end{array}$ & $\begin{array}{l}\mathrm{DR}_{v} \\
\mathrm{PO}_{v}\end{array}$ & $\begin{array}{l}\mathrm{DR}_{v} \\
\mathrm{PO}_{v}\end{array}$ & All & $\mathrm{DRv}$ \\
\hline$={ }_{v}$ & $\mathrm{PO}_{v}$ & $\mathrm{PO}_{v}$ & $\mathrm{PO}_{v}$ & $\mathrm{PO}_{v}$ & $\mathrm{PP}_{v}$ & $\mathrm{DR}_{v}$ \\
\hline
\end{tabular}

Initially, we may have enough information regarding the yolk and egg making up the region, but as a vague region starts moving, the degree of certainty of information at hand decreases and the range of the egg and the yolk start expanding. With time, the uncertainty reaches a level where distinguishing the yolk from the white becomes impossible and we end up with a scrambled egg [6. 7]. Usually, there is high probability that the object is located in a relatively small subregion of the scrambled egg. 6] uses fuzzy membership functions to represent the possibility of locating the object within the scrambled egg. For example if Tom leaves home at 8:00 AM, at 9:00 AM he may be located anywhere in a scrambled egg that may extend $100 \mathrm{kms}$ in all directions. It is much more likely however that he is still in town and even a higher probability that he is at work. 
Here, instead of taking a probabilistic approach to the problem, we propose a logic approach. This approach is nonmonotonic as it allows considering multiple possible scenarios concurrently and prefers the one best supported by available evidence. To illustrate this approach, let us consider a slightly modified version of the example in the previous paragraph.

- Tom left at 8:00 AM.

- Tom could have gone to the office.

- Tom could have gone to meet a client.

- The office called at 9:00 A.M. Tom is not in the office.

The first statement is enough to form a first scenario: a scrambled egg that is growing as a function of time in all directions at the fastest speed we could expect Tom to travel at. This is the fundamental scenario and any other scenario must be spatially constrained to the egg defined by the fundamental scenario. The second statement allows a second scenario that confines Tom to his office. This scenario cannot be valid except from the time 8:00 $+\Delta \mathrm{t} 1$, where $\Delta \mathrm{t} 1$ represents the time necessary for the scrambled egg in the fundamental scenario to reach the office. The third statement licenses us to create a third scenario placing Tom at the client's site from 8:00 $+\Delta \mathrm{t} 2$ where $\Delta \mathrm{t} 2$ is the shortest possible time to reach the client's site. The fourth statement introduces a conflict with the second scenario, forcing its retraction. Now we are left with the fundamental (first) and third scenarios. The fundamental scenario will continue to grow until we get reliable and conclusive evidence regarding Tom's location at a particular time forcing the fundamental scenario to shrink and start expanding over time again. All scenarios other than the fundamental one use persistence and causation. The fundamental scenario provides a general consistency constraint and a failsafe answer that may not be very useful.

In [16] a simplified version of this approach has been implemented to reidentify vehicles as they travel along the highway. Each highway section is equipped with inductive loop detectors that record vehicle length, lane, speed and electromagnetic signature. By forming the fundamental eggs and matching signature, length, and lane information, a large majority of vehicles has been correctly re-identified. In many cases several matches resulted for the same vehicle. In these cases, all the possible matches are kept as mutually exclusive possible scenarios.

Sometimes, it is possible to justify that the set of mutually exclusive scenarios is exhaustive (i.e. it is not possible that no new information will justify a new scenario). In these cases, the fundamental scenario is not needed. In these cases, we consider that a scrambled egg SPLIT has occurred. This scrambled egg split is very different from the SPLIT of a crisp region as defined in (11) because the regions a scrambled egg splits into do not have to be continuous, and they do not have to add up to form the original egg. Splitting a non-scrambled eggyolk region does not represent the same problem as a scrambled egg. In this case, the egg must be split the same way as for a crisp region according to 11 Note that splitting is the only case where it is possible to use EC with nonscrambled egg-yolk regions. As we split a vague region into two subregions, the 
subregions are externally connected irrespective of the boundaries. A scrambled egg region however, may split into two or more egg-yolk pairs depending upon the information obtained from the object re-identification as one or more objects may satisfy the characteristics of our object in motion. This can be problematic as re-identification splits our scrambled-egg into two objects; this implies allowing branching time which is strictly prohibited by the continuity assumptions guiding this work. We overcome this difficulty by placing each region resulting from the splitting in a separate scenario. Within each scenario, time is linear and motion of all regions is continuous. The only way to combine information from different scenarios is through the use of disjunctions.

\section{Conclusion and Ongoing Research}

We have found a methodology to modify Muller's representation crisp spatiotemporal regions to accommodate vague regions. We applied this methodology to perform reasoning using the composition tables to answer queries to obtain spatial and temporal information regarding two moving regions. We have also found a subset of the motion classes defined by Muller to suit vague regions. Current research is taking into account the composition tables and formulating general rules to reduce the size of the result of the compositions by trying to eliminate some of the results. Also, we are currently trying to answer different queries by varying the entries in the composition tables to suit the query at hand. Another open question is how to formalize the object re-identification and incorporate it into this first-order theory.

\section{References}

1. Allen, J.: Maintaining knowledge about temporal intervals. Communications of the ACM. 26 (1983) 832-843

2. Asher, N. and Vieu, L.: Towards a geometry of commonsense: A semantics and a complete axiomatisation of mereotopology. In Proceedings of the Fourteenth International Joint Conference on Artificial Intelligence (IJCAI'95)(1995)

3. Clarke, B.: A calculus of individuals based on 'connection'. Notre Dame Journal of Formal Logic. 22 (1981) 204-218

4. Cohn, N. and Gotts, N.: A theory of spatial regions with indeterminate boundaries. C. Eschenbach, C. Habel and B. Smith (eds), Topological Foundations of Cognitive Science 1994

5. Cohn, N. and Gotts, N.: The 'Egg-Yolk' representation of regions with indeterminate boundaries. In Burough, P. and Frank, A., eds. Geographical Objects with Indeterminate Boundaries, GISDATA 2(1996) 171-187.

6. Guesgen, H.: From the Egg-Yolk to the scrambled-egg theory. Proceedings of the Fifteenth Internatinal Florida Artificial Intelligence Research Symbosium (FLAIRS'2002) (2002)

7. Guesgen, H.: When regions start to move. Proceedings of the Sixteenth Internatinal Florida Artificial Intelligence Research Symbosium (FLAIRS'2003)(2003)

8. Hazarika, S., and Cohn, A.: Qualitative spatiotemporal continuity. conference on Spatial Information Theory (COSIT) (2001) 92-107. 
9. Hornsby, K. and Egenhofer, M.: Identity-based change: A foundation for knowledge representation International Journal of Geographical Information Science. 14 (2000) 207-224

10. Lehmann, F. and Cohn, A.: The EGG/YOLK reliability hierarchy: semantic data integration using sorts with prototypes. Proceedings of Conference on Information Knowledge Management. (1994)

11. Muller, P.: A qualitative theory of motion based on spatiotemporal primitives. Principles of Knowledge Representation and Reasoning (1998).

12. Muller, P.: Space-Time as a primitive for space and motion. Proceedings of FOIS, Frontiers in Artificial Intelligence Applications (1998).

13. Muller, P.: Topological spatiotemporal reasoning and representation. Computational Intelligence 18(3) (2002) 420-450.

14. Rajagopalan, R. and Kuipers, B.: Qualitative spatial reasoning about objects in motion. Proceedings of the IEEE Conference on Artificial Intelligence for Applications, vol. 46. (1994)

15. Randell, D., Cui, Z., Cohn, A.: A spatial logic based on regions and connection. Proceedings of Knowledge Representation and Reasoning, KR \& R 92 (1992) 165176.

16. Tawfik, A., Peng, A., Tabib, S. and Abdulhai, B.: Learning spatiotemporal context for vehicle reidentification. Proceedings of the 2nd IEEE International Symposium on Signal Processing and Information Technology. (2002)

17. Wolter, F. and Zakharyaschev, M.: Spatiotemporal representation and reasoning based on RCC-8. In Proceedings of the 17th Conf. on Principles of Knowledge Representation and Reasoning, KR-2000. : (2000) 3-14. 